\title{
Hadron production at LHC in dipole momentum space
}

\author{
E. A. F. Basso*, M. B. Gay Ducati* and E. G. de Oliveira ${ }^{\dagger}$ \\ *Instituto de Física, Universidade Federal do Rio Grande do Sul, Caixa Postal 15051, 91501-970 \\ - Porto Alegre, RS, Brazil \\ ${ }^{\dagger}$ Instituto de Física, Universidade de São Paulo, Caixa Postal 66318, 05314-970 São Paulo, SP, \\ Brazil
}

\begin{abstract}
.
The dipole color approach is the framework that considers the quark-antiquark pair scattering off the target. The rapidity evolution of color dipoles is given by the nonlinear Balitsky-Kovchegov (BK) equation, for which analytical solutions are not yet known. A good way to explore the asymptotic BK solutions is through the traveling wave method of QCD, that uses a correspondence between the BK evolution equation in momentum space and reaction-diffusion physics. Using the traveling wave based AGBS model for the dipole amplitude in momentum space, and within the $k_{t}$-factorization formalism, we describe the LHC data on single inclusive hadron yield for $p-p$ collisions.
\end{abstract}

PACS: $12.38 . \mathrm{Bx}, 13.60 . \mathrm{Hb}, 13.85 . \mathrm{Ni}$

\section{AGBS PARAMETRIZATION}

A parametrization of dipole-proton scattering amplitude in momentum space has been proposed by de Santana Amaral, Gay Ducati, Betemps, and Soyez (AGBS) [1]. The $F_{2}$ structure function takes the form:

$$
F_{2}\left(x, Q^{2}\right)=\frac{Q^{2} R_{p}^{2} N_{c}}{4 \pi^{2}} \int_{0}^{\infty} \frac{d k}{k} \int_{0}^{1} d z\left|\tilde{\Psi}\left(k^{2}, z ; Q^{2}\right)\right|^{2} \tilde{T}(k, Y)
$$

where now the photon wavefunction is expressed in momentum space and $\tilde{T}(k, Y)$ is the scattering amplitude in momentum space. The AGBS model analytically interpolates between the behavior of the BK scattering amplitude in the dilute regime and the saturation one, in which it behaves like

$$
N(k, Y) \stackrel{k \ll Q_{s}}{=} c-\log \left(\frac{k}{Q_{s}(Y)}\right) .
$$

The expression for the tail of the scattering amplitude reads

$$
N(k, Y) \stackrel{k \gg Q_{s}}{\approx}\left(\frac{k^{2}}{Q_{s}^{2}(Y)}\right)^{-\gamma_{c}} \log \left(\frac{k^{2}}{Q_{s}^{2}(Y)}\right) \exp \left[-\frac{\log ^{2}\left(k^{2} / Q_{s}^{2}(Y)\right)}{2 \bar{\alpha} \chi^{\prime \prime}\left(\gamma_{c}\right) Y}\right],
$$

where $\lambda=\min \bar{\alpha} \frac{\chi(\gamma)}{\gamma}=\bar{\alpha} \frac{\chi\left(\gamma_{c}\right)}{\gamma_{c}}=\bar{\alpha} \chi^{\prime}\left(\gamma_{c}\right)$ 
Interpolation in the AGBS model is done through the following expression for the scattering amplitude $\left(\rho \equiv \ln \left(k^{2} / k_{0}^{2}\right)\right)$

$$
\tilde{T}^{\mathrm{AGBS}}(\rho, Y)=L_{F}\left(1-e^{-T_{\mathrm{dil}}}\right),
$$

where

$$
\begin{gathered}
T_{\text {dil }}=\exp \left[-\gamma_{c}\left(\rho-\rho_{s}\right)-\frac{\mathscr{L}^{2}-\log ^{2}(2)}{2 \bar{\alpha} \chi^{\prime \prime}\left(\gamma_{c}\right) Y}\right], \\
\mathscr{L}=\ln \left[1+e^{\left(\rho-\rho_{s}\right)}\right] \quad \text { with } \quad Q_{S}^{2}(Y)=k_{0}^{2} e^{\lambda Y},
\end{gathered}
$$

and

$$
L_{F}=1+\ln \left[e^{\frac{1}{2}\left(\rho-\rho_{s}\right)}+e^{-\frac{1}{2}\left(\rho-\rho_{s}\right)}\right] .
$$

Such model was already used to investigate the possibility of gluon number fluctuations on the high energy evolution at HERA energies [2]. Recently it has been shown that the AGBS model describes equally well the DIS and inclusive hadron production in $p+A$ and $p+p$ collisions [3], defining the model parameters used in the present work. This was made in a new simultaneous fit to HERA data on the proton structure function [4] and RHIC data on the $p_{t}$ distribution of the produced hadrons and pions from BRAHMS and STAR collaborations [5]. This fit will be used here within the $k_{t^{-}}$ factorization formalism to describe the hadron production at LHC.

\section{HADRON PRODUCTION FROM $k_{t}$-FACTORIZATION}

To describe the single inclusive hadron production we use the $k_{t}$-factorization formalism [6], which states that the cross section for gluon production is given by

$$
\begin{aligned}
\frac{d \sigma^{A+B \rightarrow g}}{d y d^{2} p_{t} d^{2} R} & =K \frac{2}{C_{F} p_{t}^{2}} \int^{p_{t}} \frac{d^{2} k_{t}}{4} \\
& \times \int d^{2} b \alpha_{S}(Q) \varphi\left(\frac{\left|p_{t}+k_{t}\right|}{2}, x_{1} ; b\right) \varphi\left(\frac{\left|p_{t}-k_{t}\right|}{2}, x_{2} ; R-b\right),
\end{aligned}
$$

where $x_{1,2}=\left(p_{t} / \sqrt{s}\right) e^{ \pm y}$ are momentum fractions of the incoming gluons and $C_{F}=$ $\left(N_{c}^{2}-\right) / 2 N_{c}$ is the Casimir for the fundamental representation. Within this formalism we have used $K=2$.

In the large $N_{c}$ limit, the unintegrated gluon distribution in either of the two colliding hadrons can be related to the dipole scattering amplitude through

$$
\varphi(k, x ; b)=\frac{N_{c}}{2 \pi^{2} \alpha_{S}(k)} k^{2} \nabla_{k}^{2} N_{G}(k, x ; b) .
$$

The charged hadron yield, given in terms of (8), reads

$$
\frac{d N_{c h}}{d \eta d^{2} p_{t}}=\frac{h[\eta]}{\sigma_{n s d}} \int d^{2} R \int \frac{d z}{z^{2}} D_{h}\left(z=p_{t} / k_{t}, \mu\right) \frac{d \sigma^{A+B \rightarrow g}}{d y d^{2} p_{t} d^{2} R},
$$



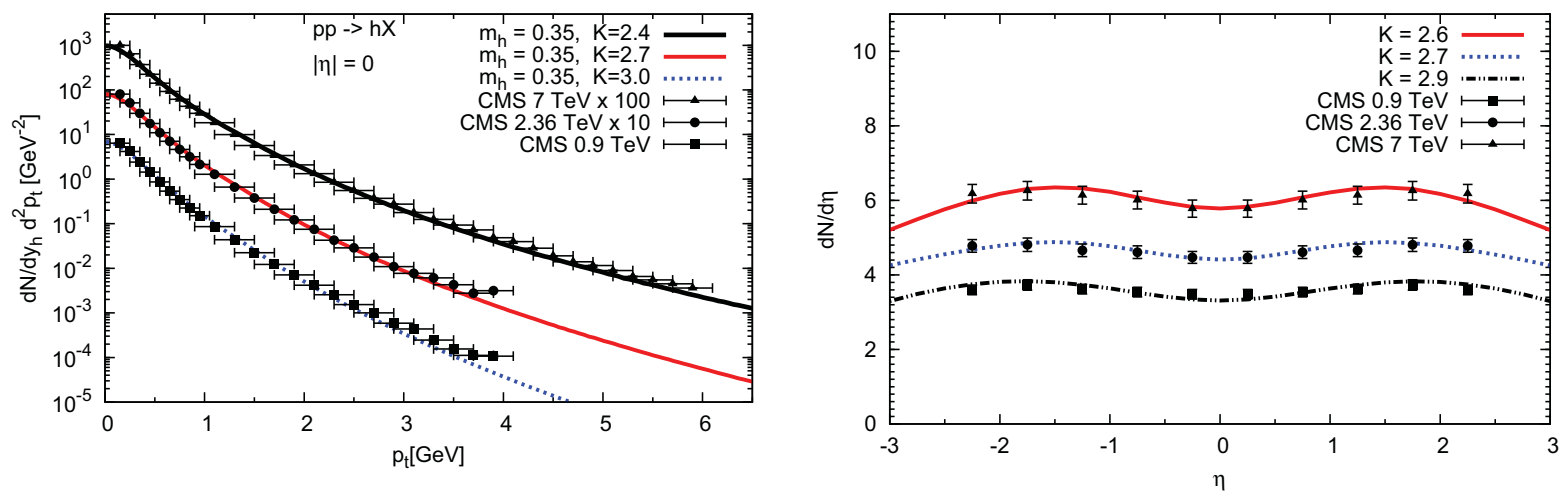

FIGURE 1. Predictions of the AGBS model to the LHC CMS charged hadron yield for $p+p$ collisions at $\sqrt{s}=0.9,2.36$ and $7 \mathrm{TeV}[9]$.

where $D_{h}\left(z=p_{t} / k_{t}, \mu\right)$ stands for the fragmentation function of the produced gluon into hadrons, for which we used the LO KKP model [7] with the scale $\mu=p_{t}$ of the hadron, and $\sigma_{n s d}$ is the non-single-diffractive cross section taken from the KMR model [8]. The model describe quite well the LHC data [9] on both the high and small $p_{t}$ regions, as seen in Figure 1. Thus, it appears as a good tool to investigate saturation physics on the $p+P b$ LHC running, where one can really consider a dilute proton interacting with the dense gluonic field of the nucleus. This will allow us to confirm whether such saturation effects are really worth to describe the small- $x$ data.

\section{ACKNOWLEDGMENTS}

This work is supported by CNPq (Brazil). E.G.O. is supported by FAPESP under contract 2011/50597-8.

\section{REFERENCES}

1. J. T. de Santana Amaral, M. B. Gay Ducati, M. A. Betemps and G. Soyez, Phys. Rev. D 76, 094018 (2007)

2. E. Basso, M. B. Gay Ducati, E. G. de Oliviera, and J. T. de Santana Amaral, Eur. Phys. J. C 58, 9 (2008); E. Basso, M. B. Gay Ducati, E. G. de Oliviera, and J. T. de Santana Amaral, Braz. J. Phys. v. 38, 483 (2008).

3. E. A. F. Basso, M. B. Gay Ducati and E. G. de Oliveira, Phys. Rev. D 84, 034024 (2011).

4. F. D. Aaron et al. (H1 and ZEUS Collaborations), JHEP 0110109 (2010).

5. I. Arsene et al. (BRAHMS Collaboration), Phys. Rev. Lett. 93, 242303 (2004); J. Adams et al. (STAR collaboration), Phys. Rev. Lett. 97, 152302 (2006).

6. M. Braun, Phys. Lett. B 483, 105 (2000); Y. V. Kovchegov and K. Tuchin, Phys. Rev. D 65, 074026 (2002).

7. B. A. Kniehl, G. Krammer and B. Pötter, Nucl. Phys. B582, 514 (2000).

8. M. G. Ryskin, A. D. Martin and V. A. Khoze, Eur. Phys. J. C 71 (2011) 1617.

9. V. Khachatryan et al. (CMS Collaboration), JHEP 0210, 041 (2010) [arXiv:hep-ex/1002.0621]; Phys. Rev. Lett. 105, 022002 (2010). 
Copyright of AIP Conference Proceedings is the property of American Institute of Physics and its content may not be copied or emailed to multiple sites or posted to a listserv without the copyright holder's express written permission. However, users may print, download, or email articles for individual use. 